\title{
Intervención educativa basado en evidencia en agentes comunitarios de salud alto andinos sobre emergencias materno-neonatal
}

\author{
Mery Luz Capcha Huamani \\ merychsalud@gmail.com \\ https://orcid.org/0000-0001-7522-5114 \\ Licenciado en enfermería \\ Universidad Nacional Autónoma de Tayacaja \\ Daniel Hernández Morillo \\ Blas Oscar Sanchez Ramos \\ oscarsanchez@unat.edu.pe \\ https://orcid.org/0000-0003-1685-0627 \\ Licenciado en enfermería \\ Universidad Nacional Autónoma de \\ Tayacaja Daniel Hernández Morillo \\ Raúl Ureta Jurado \\ raul.ureta@unh.edu.pe \\ Dirección Regional de Salud Huancavelica
}

\section{RESUMEN}

Objetivo. Determinar de qué manera la intervención educativa basado en evidencias fortalece las competencias del agente comunitario de salud alto andinos sobre emergencias materno- neonatal del Huancavelica , Perú - 2019. Métodos. Se realizó un estudio de tipo cuantitativo, diseño pre experimental con un solo grupo en agentes comunitarios de salud de la Microred de Ascensión. La muestra incluye agentes comunitarios $(\mathrm{n}=16)$; de quienes se realizó la recolección de datos sobre las competencias en emergencias materno-neonatal. Resultados. 50,0\% de agentes comunitario de salud de la Microred de Ascensión son del género masculino y femenino, respectivamente; $56,3 \%$, de 36 a 45 años y 43,8\%, de 26 a 35 años; 43,8\%, con primaria completa, y $31,3 \%$, secundaria incompleta; y un mínimo número de otro grado de instrucción. El 62,5\%, momento antes de la estrategia presentaban una competencia cognitiva en inicio sobre emergencia materno-neonatal y 37,5\%, en proceso; después de la intervención; $75,0 \%$, lograron la competencia cognitiva, y 25,0\%; en proceso. El $68,8 \%$, antes de la estrategia presentaban una competencia procedimental en inicio sobre las emergencias materno-neonatal y 31,3\%, en proceso; después de la intervención; 
$75,0 \%$, lograron la competencia procedimental, y 25,0\%; en proceso. Un 75,0\%, antes de la estrategia presentaban una competencia actitudinal en inicio sobre las emergencias materno-neonatal y 25,0\%, en proceso; después de la intervención; 93,8\%, lograron la competencia actitudinal, y 6,3\%; en proceso. Y, 56,3\%, antes de la estrategia presentaban una competencia en inicio sobre emergencia materno-neonatal y 43,8\%, en proceso; después de la intervención; 81,3\%, lograron la competencia, y 18,8\%; en proceso. Conclusiones. Las intervenciones educativas basados en evidencias lograron incrementar las competencias en manejo de emergencia materno-neonatal en agentes comunitarios de salud. Por ello, puede ser implementado en otras instituciones prestadoras de servicios de salud-IPRESS.

Palabra clave: materno-neonatal; agente comunitario; salud comunitaria. 


\title{
Evidence-based educational intervention on maternal-neonatal emergencies for high andean community health workers
}

\begin{abstract}
ABSTRAC
Objective. To determine how the evidence-based educational intervention strengthens
\end{abstract} the competencies of the community health agent on maternal and neonatal emergencies of the Ascension District - 2019. Methods A quantitative study was conducted, preexperimental design with a single group in community health agents of the Ascension Microred. The sample includes community agents $(n=16)$; of those who collected data on competencies in maternal and neonatal emergencies. Results $50.0 \%$ of community health agents of the Ascension Microred are male and female, respectively; 56.3\%, from 36 to 45 years old and $43.8 \%$, from 26 to 35 years old; $43.8 \%$, with full primary, and $31.3 \%$, incomplete secondary; and a minimum number of another degree of instruction. $62.5 \%$, just before the strategy presented a cognitive competence at the beginning on maternal-neonatal emergency and 37.5\%, in process; after the intervention; $75.0 \%$, achieved cognitive competence, and $25.0 \%$; in process. $68.8 \%$, before the strategy presented a procedural competence at the beginning on maternal-neonatal emergencies and $31.3 \%$, in process; after the intervention; $75.0 \%$, achieved procedural competence, and $25.0 \%$; in process. $75.0 \%$, prior to the strategy, presented initial attitudinal competence over maternal and neonatal emergencies and $25.0 \%$ in the process; after the intervention; $93.8 \%$, achieved attitudinal competition, and 6.3\%; in process. And, $56.3 \%$, before the strategy, presented a competition in the beginning on maternal-neonatal emergency and $43.8 \%$, in process; after the intervention; $81.3 \%$, achieved the competition, and 18.8\%; in process. Conclusions Educational interventions managed to increase the skills in maternal and neonatal emergency in community health agents. Therefore, it can be implemented in other institutions providing health services-IPRESS.

Keyword: maternal-neonatal; community agent; community health.

Artículo recibido: 05 octubre. 2021 Aceptado para publicación: 02 noviembre 2021 Correspondencia: merychsalud@gmail.com Conflictos de Interés: Ninguna que declarar 


\section{INTRODUCCION}

La participación del agente comunitario de la salud, en acciones coordinadas con los profesionales de salud se da en el sentido de reforzar la interacción del Establecimiento de Salud con las comunidades en el desarrollo sanitario, dando formación a algunos de sus miembros y hacerlos participar como Agentes de Salud Comunitarios. (Rodríguez, 2016)

Desafortunadamente, en América Latina y el Caribe, el derecho a la salud no siempre es una realidad para muchos de sus ciudadanas y ciudadanos. El acceso efectivo a la salud y la calidad de los servicios presentan grandes diferencias entre países y en su interior. Además, amplios sectores de su población enfrentan situaciones de enorme vulnerabilidad y están privados de las condiciones necesarias para una vida sana y del acceso a servicios sanitarios (Bernal, 2014)

Para lograr una mayor racionalidad y equidad en el desarrollo de los servicios de salud, hay que conceder más importancia a la salud que a la enfermedad y más a la atención de salud que a la atención médica. Sin embargo, actualmente la inversión de recursos destinados a servicios hospitalarios y especializados sigue siendo en España el 54,2\% frente la atención primaria $15,1 \%$. (Personat C , 2013)

Las muertes maternas y neonatales, como expresión máxima de las complicaciones del embarazo, parto y puerperio, aún constituyen un grave tema de salud pública. A pesar de ser un problema altamente evitable y de haber sido reducido en forma considerablemente en algunos países, en otros (y en algunas áreas vulnerables al interior de los países) se ha progresado poco, y por tanto continúa siendo una agenda inconclusa. Existen estrategias y medios que pueden ser altamente efectivos, como el conocimiento y la experiencia lo indican, y que son aplicables incluso en aquellos grupos de población que son más vulnerables y susceptibles de enfermar y morir por causas altamente evitables.(OPS, 2017)

En el Perú el agente comunitario de salud, cumple un importante papel como nexo en la relación entre la comunidad y las instituciones de salud. Sus tareas incluyen la prestación de servicios de fomento de la salud, prevención de la enfermedad, y detección y atención temprana de las enfermedades más frecuentes de los neonatos y niños. Por la cual en su propósito de fortalecer las competencias técnica de los agentes comunitarios de salud, considera de gran importancia la capacitación de los agentes comunitarios de salud y la 
familia en las prácticas clave en emergencias materno-neonatal para prevenir y disminuir la morbilidad y mortalidad de neonatos, niños y gestantes, donde el agente comunitario de salud dentro de la comunidad están en la capacidad de ofrecer atención a las madres, recién nacidos, niños y niñas, promoviendo de esta manera el acceso equitativo y universal a la atención de salud. (Rodríguez, 2016)

En este contexto el presente estudio tuvo por objetivo determinar de qué manera la intervención educativa basado en evidencias fortalece las competencias del agente comunitario de salud sobre emergencias materno- neonatal del distrito de ascensión 2019, teniendo como muestra 16 agentes comunitarios de salud activos.

\section{MATERIAL Y METODO}

Se realizó un estudio de tipo cuantitativo, diseño pre experimental con un solo grupo en agentes comunitarios de salud de la Microred de Ascensión. La muestra incluye agentes comunitarios $(n=16)$; de quienes se realizó la recolección de datos sobre las competencias en emergencias materno-neonatal.

\section{RESULTADOS}

Para la descripción e interpretación de datos obtenidos luego de la aplicación del instrumento de medición: cuestionario sobre competencias en emergencias básicas en materno-neonatal en los agentes comunitarios de salud de la Microred de Ascensión, Huancavelica.

Se organizó los datos recolectados para la representación de los mismos haciendo uso del paquete estadístico IBM SPSS Statistics para Windows Vers. 25.0 y Microsoft OfficeExcel 2016; tomando en cuenta que los datos obtenidos son variables cualitativas y cuantitativas

Tabla $\mathbf{N}^{\circ}$ 01: Característica sociodemográfica del agente comunitario de salud en la Microred de Ascensión, Huancavelica

\begin{tabular}{llll}
\hline \multicolumn{2}{l}{ Características sociodemográfica } & $\mathbf{f i}$ & $\mathbf{f \%}$ \\
\hline \multirow{2}{*}{ Género } & Masculino & 8 & 50.0 \\
Total & Femenino & 8 & 50.0 \\
Edad & & $\mathbf{1 6}$ & $\mathbf{1 0 0 . 0}$ \\
Total & 26 a 35 años & 7 & 43.8 \\
& 36 a 45 años & 9 & 56.3 \\
\multirow{3}{*}{ Grado de instrucción } & & $\mathbf{1 6}$ & $\mathbf{1 0 0 . 0}$ \\
& Primaria incompleta & 2 & 12.5 \\
& Primaria completa & 1 & 6.3 \\
Total & Secundaria incompleta & 5 & 31.3 \\
\hline & Secundaria completa & 7 & 43.8 \\
& Superior incompleto & 1 & 6.3 \\
\hline
\end{tabular}

Fuente: Cuestionario aplicado, 2019 
En la Tabla y Gráfico No 01 se observa que 50,0\% de agentes comunitario de salud de la Microred de Ascensión son del género masculino y femenino, respectivamente; 56,3\%, de 36 a 45 años y 43,8\%, de 26 a 35 años; 43,8\%, con primaria completa, y 31,3\%, secundaria incompleta; y un mínimo número de otro grado de instrucción.

Tabla $\mathbf{N}^{\circ}$ 02: Competencia cognitiva sobre emergencia materno-neonatal en agente comunitario de salud en la Microred de Ascensión, Huancavelica.

\begin{tabular}{lcc|cc}
\hline \multirow{2}{*}{$\begin{array}{c}\text { Competencia cognitiva en } \\
\text { emergencia materno-neonatal }\end{array}$} & \multicolumn{4}{c}{ Momento de medición } \\
\cline { 2 - 6 } & Antes & $\mathbf{f \%}$ & fi & Después \\
\hline Logrado & 0 & 0.0 & 12 & 75.0 \\
En proceso & 6 & 37.5 & 4 & 25.0 \\
En inicio & 10 & 62.5 & 0 & 0.0 \\
Total & $\mathbf{1 6}$ & $\mathbf{1 0 0 . 0}$ & $\mathbf{1 6}$ & $\mathbf{1 0 0 . 0}$ \\
\hline
\end{tabular}

$$
t_{k}=-7,704 \quad t_{T(0,025 ; 15)}=2,13 \quad r_{(n-1)}=15 \quad p_{\text {value }}=, 000
$$

En la Tabla y Gráfico $N^{\circ} 02$ se observa que $62,5 \%$ de agentes comunitario de salud de la Microred de Ascensión momento antes de la estrategia presentaban una competencia cognitiva en inicio sobre emergencia materno-neonatal y 37,5\%, en proceso; después de la intervención; 75,0\%, lograron la competencia cognitiva, y 25,0\%; en proceso.

Tabla No 03: Competencia procedimental sobre emergencia materno-neonatal en agente comunitario de salud en la Microred de Ascensión, Huancavelica.

\begin{tabular}{|c|c|c|c|c|}
\hline \multirow{3}{*}{$\begin{array}{l}\text { Competencia procedimental en } \\
\text { emergencia materno-neonatal }\end{array}$} & \multicolumn{4}{|c|}{ Momento de medición } \\
\hline & \multicolumn{2}{|c|}{ Antes } & \multicolumn{2}{|c|}{ Después } \\
\hline & fi & $\mathrm{f} \%$ & $\mathbf{f i}$ & $\mathbf{f \%}$ \\
\hline Logrado & 0 & 0.0 & 12 & 75.0 \\
\hline En proceso & 5 & 31.3 & 4 & 25.0 \\
\hline En inicio & 11 & 68.8 & 0 & 0.0 \\
\hline Total & 16 & 100.0 & 16 & 100.0 \\
\hline
\end{tabular}

Fuente: Cuestionario aplicado, 2019
$t_{k}=-11,165$
$t_{T}(0,025 ; 15)=2,13$
$r_{(n-1)}=15$
$p_{\text {value }}=, 000$

En la Tabla y Gráfico $\mathrm{N}^{\mathrm{o}} 03$ se observa que $68,8 \%$ de agentes comunitarios de salud de la Microred de Ascensión momento antes de la estrategia presentaban una competencia procedimental en inicio sobre las emergencias materno-neonatal y 31,3\%, en proceso; después de la intervención; 75,0\%, lograron la competencia procedimental, y 25,0\%; en proceso. 
Tabla No 04: Competencia actitudinal sobre emergencia materno-neonatal en agentes comunitario de salud en la Microred de Ascensión, Huancavelica.

\begin{tabular}{lcc|cc}
\hline \multirow{2}{*}{$\begin{array}{c}\text { Competencia actitudinal en } \\
\text { emergencia materno-neonatal }\end{array}$} & \multicolumn{2}{c}{ Momento de medición } \\
\cline { 2 - 5 } & Antes & \multicolumn{2}{c}{ Después } \\
\hline Logrado & $\mathbf{f i}$ & $\mathbf{f \%}$ & $\mathbf{f i}$ & $\mathbf{f \%}$ \\
En proceso & 0 & 0.0 & 15 & 93.8 \\
En inicio & 4 & 25.0 & 1 & 6.3 \\
Total & 12 & 75.0 & 0 & 0.0 \\
\hline & $\mathbf{1 6}$ & $\mathbf{1 0 0 . 0}$ & $\mathbf{1 6}$ & $\mathbf{1 0 0 . 0}$ \\
\hline
\end{tabular}

Fuente: Cuestionario aplicado, 2019

$t_{k}=-15,504 \quad t_{T(0,025 ; 15)}=2,13 \quad r_{(n-1)}=15 \quad p_{\text {value }}=, 000$

En la Tabla y Gráfico № 04 se observa que 75,0\% de agentes comunitarios de salud de la Microred de Ascensión momento antes de la estrategia presentaban una competencia actitudinal en inicio sobre las emergencias materno-neonatal y 25,0\%, en proceso; después de la intervención; 93,8\%, lograron la competencia actitudinal, y 6,3\%; en proceso.

Tabla No 05: Competencia general sobre emergencia materno-neonatal en agentes comunitario de salud en la Microred de Ascensión, Huancavelica.

\begin{tabular}{lcc|cc}
\hline \multirow{2}{*}{$\begin{array}{c}\text { Competencia general en } \\
\text { emergencia materno-neonatal }\end{array}$} & \multicolumn{4}{c}{ Momento de medición } \\
\cline { 2 - 5 } & fi & $\mathbf{f} \%$ & $\mathbf{f i}$ & fespués \\
\hline Logrado & 0 & 0.0 & 13 & 81.3 \\
En proceso & 7 & 43.8 & 3 & 18.8 \\
En inicio & 9 & 56.3 & 0 & 0.0 \\
Total & $\mathbf{1 6}$ & $\mathbf{1 0 0 . 0}$ & $\mathbf{1 6}$ & $\mathbf{1 0 0 . 0}$ \\
\hline
\end{tabular}

Fuente: Cuestionario aplicado, 2019

$t_{k}=-14,598 \quad t_{T(0,025 ; 15)}=2,13 \quad r_{(n-1)}=15 \quad p_{\text {value }}=, 000$

En la Tabla y Gráfico № 05 se observa que 56,3\% de agentes comunitarios de salud de

la Microred de Ascensión momento antes de la estrategia presentaban una competencia en inicio sobre emergencia materno-neonatal y 43,8\%, en proceso; después de la intervención; 81,3\%, lograron la competencia, y 18,8\%; en proceso.

\section{DISCUSION DE RESULTADOS}

El resultado hallado se observa que 62,5\% de agentes comunitario de salud de la Microred de Ascensión antes de la estrategia presentaban una competencia cognitiva en inicio sobre emergencia materno-neonatal y 37,5\%, en proceso; después de la intervención; 75,0\%, lograron la competencia cognitiva, y 25,0\%; en proceso (Tabla $\mathrm{N}^{\circ} 02$ ). $68,8 \%$ de agentes comunitarios de salud de la Microred de Ascensión momento antes de la estrategia 
presentaban una competencia procedimental en inicio sobre las emergencias maternoneonatal y 31,3\%, en proceso; después de la intervención; 75,0\%, lograron la competencia procedimental, y 25,0\%; en proceso (Tabla $\mathrm{N}^{\circ} 03$ ). Un 75,0\% de agentes comunitarios de salud de la Microred de Ascensión momento antes de la estrategia presentaban una competencia actitudinal en inicio sobre las emergencias materno-neonatal y 25,0\%, en proceso; después de la intervención; 93,8\%, lograron la competencia actitudinal, y 6,3\%; en proceso (Tabla $\mathrm{N}^{\circ}$ 04). 56,3\% de agentes comunitarios de salud de la Microred de Ascensión momento antes de la estrategia presentaban una competencia en inicio sobre emergencia materno-neonatal y 43,8\%, en proceso; después de la intervención; $81,3 \%$, lograron la competencia, y $18,8 \%$; en proceso. (Tabla $\mathrm{N}^{\circ} 05$ )

Es importante destacar lo publicado por Personat Celia. 2013, Brasil; quienes señalan que el Agente Comunitario de Salud tiene el papel mediador, promotor y educador, y como su actividad en la comunidad puede transformar la salud de sus gentes. Su presencia en los sistemas de salud de los países o en organizaciones no gubernamentales no son sólo garantía de mejora de salud y del entorno social en las comunidades, sino también hay evidencia de que sus esfuerzos consiguen disminuir el gasto sanitario. Tal vez sea un sujeto transformador.

Así mismo, es importante destacar lo publicado por Pizon, D, Et al. 2015, Colombia, quien refiere que Se identificaron actividades de promoción y prevención para malaria, adherencia a la lactancia materna, cuidados del recién nacido, cuidados y soporte para el momento del parto. En todos los escenarios se demostró efectividad de la inclusión del trabajador comunitario en los desenlaces de adherencia y reducción de mortalidad materna e infantil. La inclusión de los trabajadores comunitarios es efectiva en la reducción de la mortalidad materna e infantil en países de bajos y medianos ingresos. Además de ello, mejora el acceso y la cobertura a los servicios de salud de poblaciones vulnerables.

Por otro lado, es importante señalar lo publicado por Hernandez, V, et al. 2015 Lima, donde considera que las intervenciones educación materna en salud y cuidado en casa de madres y neonatos estuvieron asociadas con disminución de mortalidad neonatal en la mitad de los seis estudios de cada grupo. La suplementación materna con multimicronutrientes, el cuidado de madre canguro y la suplementación materna prenatal 
con vitamina A no disminuyeron la mortalidad neonatal. Pocas intervenciones comunitarias heterogéneas han demostrado disminuir la mortalidad neonatal.

Es importante señalar como parte del estudio es que a mortalidad infantil y la morbilidad de los niños en el Perú han venido disminuyendo a través de los años, un aspecto importante para obtener estos resultados sanitarios ha sido el progreso de fortalecer sus capacidades desde el nivel local, promoviendo el cuidado y vigilancia de gestantes, así como su articulación con los servicios de salud, incorporando pautas para la vigilancia de la salud materna y perinatal, desde los caseríos, vinculando y animándolos a que conformen su propia red zonal, constituye una de las principales acciones de la salud pública más costosa y efectiva para disminuir el riesgo de enfermar y morir en la población materno-infantil; asegura la protección oportuna de acuerdo con las emergencias materno neonatales, constituyendo un derecho para todas madres y niños/niñas, especialmente para beneficiar a un sector específico de la población más vulnerable, excluidos, pobres y pobres extremos.

\section{CONCLUSIONES}

Se concluye que existe evidencia estadística suficiente para afirmar que la competencia alcanzada por el grupo experimental antes difiere con la puntuación alcanzada después. La magnitud de significancia estadísticas de los resultados se ve con la Sig.=pvalue < 0.05; en la que implica: Sig. (bilateral) $=, 000$; es decir; existe diferencia estadística muy significativa entre los resultados o puntuaciones alcanzados del grupo experimental antes y después. Es decir; que la competencia en emergencia materno-neonatal alcanzada momento antes en los agentes comunitarios de salud que son del grupo experimental difiere con el logrado después de la intervención.

\section{REFERENCIAS BIBLIOGRAFICAS}

Rodriguez Salvador. Ser Agente Comunitario de Salud en una Comunidad Periurbana de la ciudad de Trujillo. Peru 2016.

Bernal, et al. Innovación social en salud. Chile. 2014;

Personat C. Titol: Agente Comunitario de Salud, sujeto transformador. Brasil. 2013;

Organizacion Panamericana de la Salud, Mortalidad Materna y Neonatal en ALC y Estrategias de Reduccion. Chile 2017.

Ministerio de Salud. Programa Presupuestal 0002, Salud Materno Neonatal. Peru 2018. 
Hernández A v., Pasupuleti V, Benites-Zapata V, Velásquez-Hurtado E, Loyola-Romaní J, Rodríguez-Calviño Y, et al. Revisión sistemática sobre la efectividad de intervenciones basadas en la comunidad en la disminución de la mortalidad neonatal. Perú. 24 de septiembre de 2015;

Ministerio de Salud, Documento Tecnico: Orientacion para el fortalecimiento de la labor del Agente Comunitario de Salud. Peru 2014.

Ministerio de Economia y Finanzas, Instituto Nacional de Estadistica e Informatica, Peru.2018.

Ministerio de Salud, Direccion Regional de Salud, Huancavelica. Perú 2015.

Ministerio de Salud, His Report. Ascension, Huancavelica. Peru, 2018.pdf.

Cornejo MAV. Congreso de la Republica, Poder Legislativo. Constitucion Politica del Perú. 2018;

Ministerio de Salud, Documento Tecnico: Preparando al Agente Comunitario de Salud para el Cuidado Integral de la Salud y Nutricion de las Gestantes y de las Niñas y Niños Menores de 5 años.(El manual del Agente Comunitario de Salud). Peru 2009.

Ñamendy SS, Traña EJR. Evaluación de las estrategias Plan de Parto en la reducción de la mortalidad materna perinatal, centro de Salud Héroes y Mártires de Nueva Segovia, Municipio de Mozonte, Nueva Segovia. 2010;

Pinzón Flórez CE, Díaz-Quijano DM, Yáñez Álvarez I, Mesa DC. Effectiveness of community workers on preventive measures to maternal and child health in low and middle income countries: systematic review of the literature. salud uninorte. 1 de mayo de 2015;31(2):309-28.

Canever et al. - Percepções das agentes comunitárias de saúde sobre.pdf.

Brandt et al. - 2019 - Maternal health training priorities for nursing an.pdf.

Llumpo VR. Fomentando conocimiento de los Agentes Comunitarios en Salud Materno perinatal, Centro poblado Chontayocc. Huaraz, Peru. 2018;

Bonifacio Aliaga, Liz Zulema. Factores Determinantes Relacionados con Funcionamiento de Centros de Promocion, Vigilancia Comunal del Cuidado Integral de Madre y Niño Pasco 2014. 
Gonzales Morales. Tipo de Motivacion como Factor Relacionado al Conocimiento en los Agentes Comunitarios de la Red de Salud San Juan de Miraflores-Villa Maria del Triunfo. 2017.

Nola Pender, Teoria de Promocion de la Salud.2015.

López JMT. Intervención Educativa, Intervención Pedagógica y Educación: La Mirada Pedagógica. Madrid. 2011;

Piedrahita MVA, Gómez MCA, Mendoza MÁG, Loaiza FR, Gallón H, Kennedy I. Intervención, mediación pedagógica y los usos del texto escolar. Colombia. 2015;

Gómez MTO. Intervencion Educativa en Salud Familiar. Perú. 2013;

Acero Valdez. Nivel de Aplicación de la Estrategia de Sectorizacion en Promocion de la Salud, segun percepcion de los Actores Sociales de los Establecimientos de la Red de Salud Huanuco. Perú. 2016;

Palomino Vega CP. Participacion de los Agentes Comunitarios de Salud del Programa Municipios y Comunidades Saludables de la Micro Red de Salud TiabayaArequipa. 2017;

Ruiz DOC. Hospital Maria Auxiliadora. Guias de practica clinica para la atencion de las emergencias obstetricas. Peru. 2012;

Ministerio de la Salud. Guias de Practica Clinica Para la Atencion de Emergencias Obstetricas segun Nivel de Capacidad Resolutiva. Perú, 2012. 